ARTICLE

Received 27 Sep 2012 | Accepted 17 Apr 2013 | Published 21 May 2013

DOI: $10.1038 /$ ncomms 2900

\title{
Assembly of a $\pi-\pi$ stack of ligands in the binding site of an acetylcholine-binding protein
}

\author{
Mariano Stornaiuolo1, Gerdien E. De Kloe ${ }^{2}$, Prakash Rucktooa', Alexander Fish', René van Elk³, \\ Ewald S. Edink ${ }^{2}$, Daniel Bertrand ${ }^{4}$, August B. Smit ${ }^{3}$, Iwan J. P. de Esch ${ }^{2}$ \& Titia K. Sixma ${ }^{1}$
}

Acetylcholine-binding protein is a water-soluble homologue of the extracellular ligandbinding domain of cys-loop receptors. It is used as a structurally accessible prototype for studying ligand binding to these pharmaceutically important pentameric ion channels, in particular to nicotinic acetylcholine receptors, due to conserved binding site residues present at the interface between two subunits. Here we report that an aromatic conjugated small molecule binds acetylcholine-binding protein in an ordered $\pi-\pi$ stack of three identical molecules per binding site, two parallel and one antiparallel. Acetylcholine-binding protein stabilizes the assembly of the stack by aromatic contacts. Thanks to the plasticity of its ligand-binding site, acetylcholine-binding protein can accommodate the formation of aromatic stacks of different size by simple loop repositioning and minimal adjustment of the interactions. This type of supramolecular binding provides a novel paradigm in drug design.

\footnotetext{
${ }^{1}$ Division of Biochemistry and Center for Biomedical Genetics, Netherlands Cancer Institute, Plesmanlaan 121, 1066 CX Amsterdam, The Netherlands. 2 Division of Medicinal Chemistry, Faculty of Sciences, Amsterdam Institute for Molecules, Medicines and Systems, VU University Amsterdam, De Boelelaan 1083, 1081 HV Amsterdam, The Netherlands. ${ }^{3}$ Department of Molecular and Cellular Neurobiology, Center for Neurogenomics and Cognitive Research, VU University, 1081 HV Amsterdam, The Netherlands. ${ }^{4}$ HiQScreenSàrl, 6, rue de Compois, 1222 Vésenaz, Geneva, Switzerland. Correspondence and requests for materials should be addressed to T.K.S. (email: t.sixma@nki.nl).
} 
S creening compound libraries for biologically active molecules results in hit structures considered to be interesting starting points for drug design. Although it is often assumed that a single small drug molecule interacts with one proteinbinding site, compounds binding to target proteins with higher stoichiometry can be found during library screening ${ }^{1}$. Here, we show an unexpected ligand interaction where three identical molecules interact within a single binding site. The binding of preorganized and/or in situ-organizing small binder molecules was shown to happen on the surface of proteins but examples of supramolecular assembly happening at the canonical-binding site of a target are still rare ${ }^{2,3}$. Recently two molecules of the IRE1 inhibitor quercetin were found binding at the dimer interface of the target and proved to induce its dimerization ${ }^{4}$ (PDB: 3LJ0). An example of ligand supramolecular assembly was described also for a flavine-binding enzyme in complex with the cofactors FMN and $\mathrm{NADH}$ at the surface of the protein ${ }^{5}$ (PDB: 2VZH, 2VZJ).

Cys-loop receptors are important ligand-gated ion channels in the central and peripheral nervous systems ${ }^{6-10}$. Members include the nicotinic acetylcholine receptors (nAChR), GABA receptors, $5 \mathrm{HT}_{3}$ serotonin and glycine receptors, and all

\section{Table 1 | Chemical formula, numbering and IUPAC name of the fragments.}

2-(1-Methylimidazol-2-yl)-4,6-dipyridine

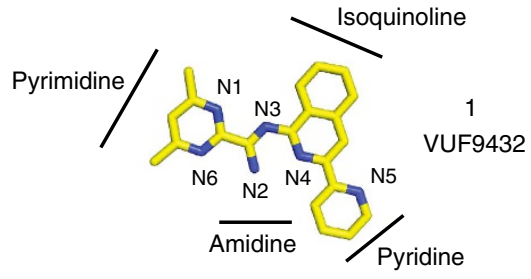

b

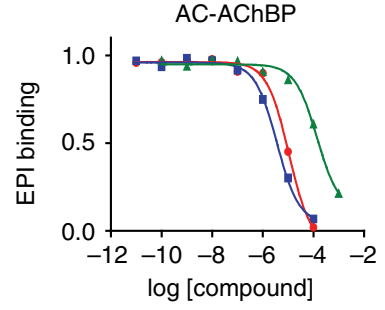

pKi (nicotine $)=5.48 \pm 0.09$ pKi (Acethylcholine $)=3.96 \pm 0.03$ pKi $($ VUF9432 $)=4.96 \pm 0.03$
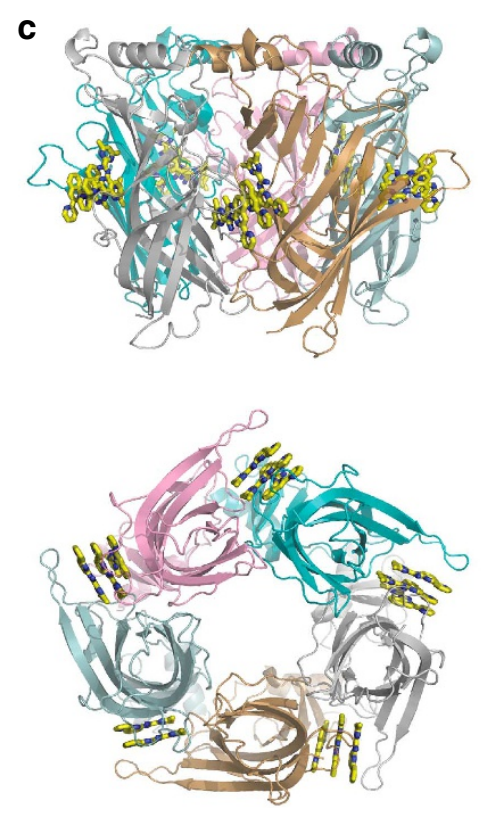

d

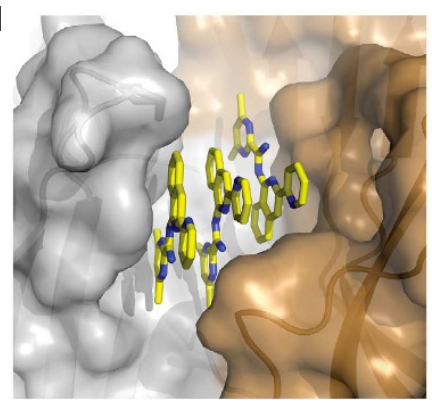

e
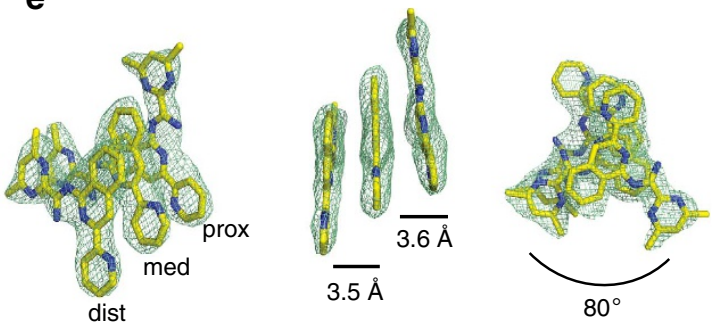

Figure 1 | VUF9432 binds as a triple stack to Ac-AChBP. (a) Chemical structure of VUF9432 (carbon atoms in yellow, nitrogen atoms in blue). (b) Displacement of radio-labelled epibatidine (EPI) by VUF9432 (red curves), nicotine (blue curves) and acetylcholine (green curves) on Ac-AChBP (purified proteins). Data are the mean \pm s.e.m. of three experiments and are reported below the panel. (c) Side and bottom side view of Ac-AChBPVUF9432 complex structure, showing VUF9432 molecules (yellow sticks) in the five binding sites. (d) Protomer-protomer interfaces (surface representation) of Ac-AChBP-VUF9432 complex. Principal side is depicted in silver and complementary side in sand colours. Ligand-binding site are shown in transparency. (e) Electron density map displaying VUF9432 molecules in the ligand-binding site formed by subunit A and B (experimental density contoured at $1 \sigma$ ), different orientation of the stacking molecules are shown together with the nomenclature used to distinguish the three molecules in the text. Intermolecular distances and angles are depicted as lines between the planes. dist, distal; med, medial; prox, proximal. 
important pharmacological targets for various diseases and conditions ${ }^{10}$. nAChRs are targets for the endogenous neurotransmitter acetylcholine and for a series of compounds aimed at the treatment of cognitive decline in Alzheimer's disease, certain forms of epilepsy ${ }^{11}$ or nicotine addiction ${ }^{12}$.

In the homo- or heteropentameric ion channels of the cys-loop receptor family, an extracellular ligand-binding site, is located at the interface between a 'principal' and a 'complementary' subunit, each of which contributes three loops (A-C and D-F, respectively) to the binding ${ }^{6-8,13}$. Agonist binding triggers conformational changes in cys-loop receptors and leads to opening of the channel and a flux of ions through the channel pore $^{14}$. Ligand-binding sites in cys-loop receptors are highly plastic, allowing binding of various molecules ranging from small neurotransmitters all the way to large peptide neurotoxins, such as conotoxins and snake toxin ${ }^{6-10}$. Flexibility for different-sized ligands is generated by the positioning of loop $\mathrm{C}$ that contributes two conserved tyrosines and a vicinal disulphide bridge to the ligand-binding site.

Most high-resolution structural information on cys-loop receptor-ligand interactions is available from a molluscan acetylcholine-binding protein $(\mathrm{AChBP})^{15-18}$. AChBP structurally resembles the ligand-binding domains of cys-loop receptors, but lacks a transmembrane domain ${ }^{16}$. Their ligand-binding site resembles the $\alpha 7$ subtype of the nAChRs most (20-24\% sequence identity) and their high-resolution crystal structures ${ }^{16-23}$ reveal a structural fold closely related to cys-loop receptors as well as to their bacterial homologs ${ }^{24-29}$.

Here we show that AChBP binds an ordered aromatic $\pi-\pi$ stack of three identical ligands. The high-resolution X-ray crystal structure of this molecule in complex with Aplysia californica (Ac)-AChBP reveals a new example of protein-ligand interaction controlled by supramolecular ligand assembly. The nature of the binding mode, the analysis of protein residues contributing to the stabilization of the stack and the kinetics of the binding events are described. Finally, we identify acridine orange (AO) as a ligand with similar binding that could potentially be used as competitive inhibitor for $\alpha 7 \mathrm{nAChR}$.

\section{Results}

Identification of VUF9432. A fragment ${ }^{30,31}$ screening assay using online fluorescence enhancement led to the identification of fragments 2-6 (Table 1) as hits for AChBP (refs 32,33). In a subsequent analogue screening, VUF9432 (1), (IUPAC name: 4,6-dimethyl- $N$-(3-(pyridin-2-yl)isoquinoline1-yl)-pyrimidine-2-carboximidamide) (Fig. 1a) was shown to have micromolar affinity for AChBP. VUF9432 was originally synthesized as a copper-dependent antimycoplasmal agent active against Mycoplasma gallisepticum proliferation and later identified in a screen as ligand for adenosine $\mathrm{A}_{3}$ receptors ${ }^{34}$.

Binding affinities of VUF9432 for Ac-AChBP and $\alpha 7 \mathrm{nAChR}$ were measured in a radioligand displacement assay with $\left[{ }^{3} \mathrm{H}\right]$ epibatidine and $\left[{ }^{3} \mathrm{H}\right]$ methyllycaconitine (MLA) as displaceable ligands for AChBPs (Fig. 1b) and $\alpha 7 \mathrm{nAChR}$ (Supplementary Fig. S1), respectively. VUF9432 behaves as competitive binder for $\mathrm{AChBP}$, showing a $\mathrm{pKi}$ value of $4.96 \pm 0.03$ for Ac-AChBP. The compound also displays some binding to $\alpha 7 \mathrm{nAChR}$ (pKi around five) but the radioligand is not fully displaced at the highest concentration tested, possibly due to low solubility of the compound under the assay conditions.

In contrast to typical nAChR targeting molecules, VUF9432 lacks the canonical cation center involved in cation- $\pi$ interactions with aromatic residues in the binding site ${ }^{13,14}$. The binding mode, interaction of VUF9432 to Ac-AChBP, was investigated by cocrystallization trials and X-ray analysis.
Table 2 | Data collection and refinement statistics.

\begin{tabular}{|c|c|}
\hline & AChBP-VUF9432 \\
\hline Data collection & PX1 (SLS) \\
\hline Space group & P21 \\
\hline \multicolumn{2}{|l|}{ Cell dimensions } \\
\hline$a, b, c(\AA)$ & $80.73,78.33,106.53$ \\
\hline$\alpha, \beta, \gamma\left(^{\circ}\right)$ & $90.00,102.67,90.00$ \\
\hline Resolution $(\AA)$ & $43.30-2.4(2.53-2.4)^{\star}$ \\
\hline Rmerge & $12.9(76.1)$ \\
\hline$|/ \sigma|$ & $5.9(1.8)$ \\
\hline Completeness (\%) & $93.0(92.6)$ \\
\hline Redundancy & 2.4 \\
\hline \multicolumn{2}{|l|}{ Refinement } \\
\hline Resolution $(\AA)$ & $41.26-2.4(2.53-2.4)$ \\
\hline No. of reflections & 44,879 \\
\hline Rwork/Rfree & $0.21 / 0.25$ \\
\hline \multicolumn{2}{|l|}{ No. of atoms } \\
\hline Protein & 8,188 \\
\hline Ligand/ion & 384 \\
\hline Water & 129 \\
\hline \multicolumn{2}{|l|}{ B-factors } \\
\hline Protein & 36.80 \\
\hline Ligand/ion & 63.48 \\
\hline Water & 27.41 \\
\hline \multicolumn{2}{|l|}{ R.m.s deviations } \\
\hline Bond lengths $(\AA)$ & 0.009 \\
\hline Bond angles $\left({ }^{\circ}\right)$ & 1.315 \\
\hline
\end{tabular}

VUF9432 binds AChBP in a triple stacked configuration. The 2.4- $\AA$ crystal structure of the complex between Ac-AChBP and VUF9432 revealed the unexpected presence of three VUF9432 molecules in four out of the five ligand-binding sites in the pentamer (the fifth site is discussed separately below) (Fig. 1c,d). Careful refinement of the protein and the asymmetric shape of the VUF9432 molecule allowed the unambiguous fitting into the electron densities (Fig. 1e and Supplementary Fig. S2), resulting in a refined structure with $R=21 \% / R_{\text {free }}=25 \%$ and excellent stereochemistry (Table 2).

Within each ligand-binding site, the three conjugated molecules of VUF9432 stack on each other (Fig. 1e) in a flat planar configuration. The three molecules are organized in a columnar stack, with an off-centred parallel displaced geometry. The distal molecule of VUF9432, closest to the C-loop and the medial one (Fig. 1e) are identically oriented with the pyrimidine rings pointing towards the inner part of the ligand-binding site, whereas the proximal molecule is twofold rotated relative to the other VUF9432 molecules. The distance between the medial molecule and the proximal and distal ones are 3.5 and $3.6 \AA$, respectively, consistent with previously measured $\pi-\pi$ stacking distances between aromatic molecules ${ }^{35}$. The angle between the planes is at most $3.8 \pm 0.8^{\circ}$ and $6.2 \pm 2.1^{\circ}$, due to slight tilting of the medial and proximal molecules towards the distal one, respectively, confirming the almost perfectly parallel displaced orientation (Fig. 1e). The proximal and distal copies of the molecule occupy slightly variable tilt angles within these planes, and hence have somewhat higher average B factors $(58.4 \pm 10.23$, $60.28 \pm 10.16$ and $74.45 \pm 11.8 \AA^{2}$ for the medial, proximal and distal copy of VUF9432, respectively). Nevertheless it is clear that all three sites are fully occupied and the excellent quality of the electron density of the C-loop indicates full occupancy of these ligands. 
a
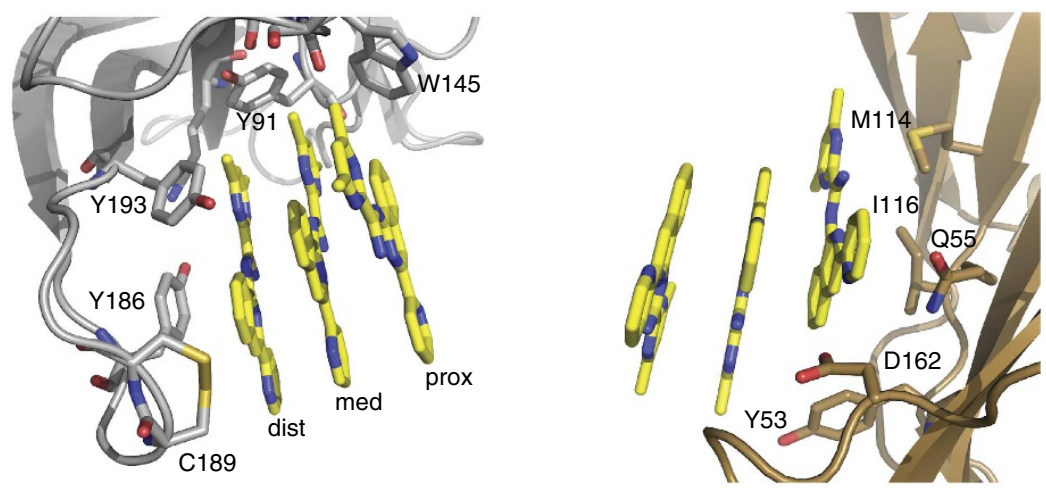

b

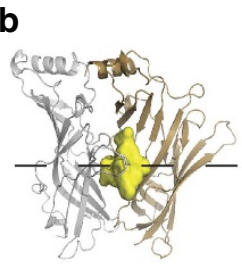

VUF9432

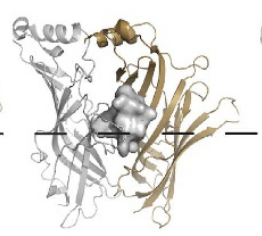

Iml

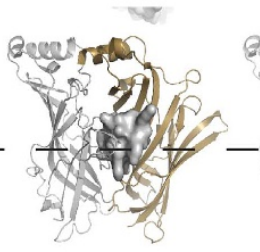

PnIA

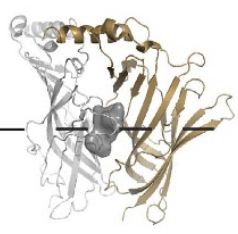

MLA
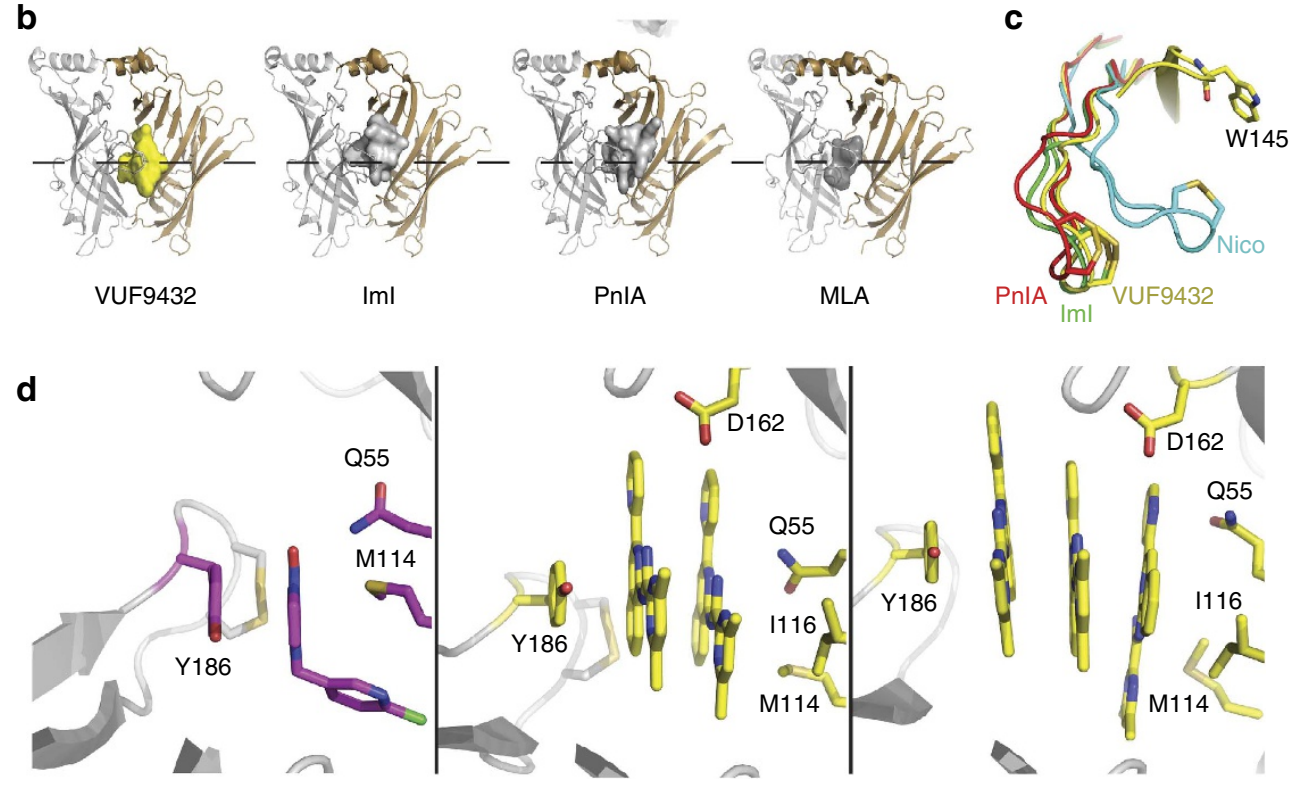

Figure 2 | Details of VUF9432 binding to Ac-AChBP. (a) Ball-and-stick representation showing Ac-AChBP residues on principal and complementary subunits interacting with VUF9432 (colours as in Fig. 1). The triple stack is held up like a row of books by Y186, Q55 and M114. Edge interactions with aromatic residues, D162 and the vicinal disulphide are indicated. (b) Protomer-protomer interfaces of VUF9432-Ac-AChBP structure illustrating how VUF9432 (surface, yellow) is occupying a lower part of the ligand-binding site if compared with the toxin IMI (surface, grey), PnIA (surface, grey) and MLA (surface, grey). (c) Comparison of C-loop opening in AChBP- nicotine (cyan), AChBP-PnIA (red), AChBP-IMI (green) and AChBP-VUF9432 (yellow) complex. Position assumed by the C-loop after VUF9432 binding resembles that in conotoxins. (d) Comparison of binding modes of Imidacloprid (magenta) and VUF9432 (yellow) to AChBP showing the two ligands establishing similar interactions with the ligand-binding site. Repositioning of the C-loop provides the space for either one, two or three flat aromatic moieties.

The stack of aromatic VUF9432 molecules is held in place like a pile of books, at one end by the stacking of well-conserved principal face residue Y186 (Fig. 2a), which has an off-centred displaced parallel $\pi-\pi$ interaction with the distal molecule, tilted at $12.3 \pm 2.4^{\circ}$. At the other end the stack is positioned through van der Waals interactions with complementary side residues Q55 and M114. The side chain of I116 is positioned towards the stack, but does not interact with the proximal molecule.

Aromatic groups on the principal (Y91, W145, Y193) and complementary faces (Y53) stabilize the sides of the stack of ligands via edge-to-face interactions, establishing $\mathrm{T}$-shaped aromatic contacts with the molecules (Fig. 2a). Finally, the F-loop D162 and the vicinal disulphide in the C-loop also interact with the sides of the stacked VUF9432 molecules.

Taken together, the three stacked molecules of VUF9432 occupy a large volume and bury a substantial surface area in AChBP $\left(959.9 \pm 4.8 \AA^{2}\right)$ comparable with the area buried by conotoxins IMI ${ }^{20}$ (PDB:2C9T) and PnIA ${ }^{19}$ (PDB:2BR8) (1164 $\AA^{2}$ and $1468 \AA^{2}$, respectively). However, the position of VUF9432 in the binding site is different, as can be seen in Fig. 2b. The space occupied by the conotoxins is above the midline of the pentamer, away from the transmembrane domain in the cys-loop receptors, whereas the VUF9432 molecules are placed lower, close to the membrane, not far from the binding site for MLA (ref. 36). However, MLA (PDB:2BYR) is in a different site closer to the five-fold axis than VUF9432, which uses a lower, more external cavity (Fig. 2b). It does not address the so-called 'lobeline pocket' and Y91 is in the g-conformation, interacting with $S 144^{37}$. The combination of this novel position and slightly smaller buried surface area result in a C-loop position that is similar to that for the conotoxins, in its most open state (Supplementary Fig. S3).

In one of the five ligand-binding sites only two molecules of VUF9432 are bound, positioned in parallel manner, similarly to the medial and the distal copy in the other ligand-binding sites (Fig. 3a,b). This state is most likely stabilized by crystal contacts (Fig. 3f). Although the double stack is tilted $15^{\circ}$ relative to the triple stack in the other binding sites (Fig. 3c), the direct contacts are retained. Thus, the stack is upheld by Y186 at one end and 


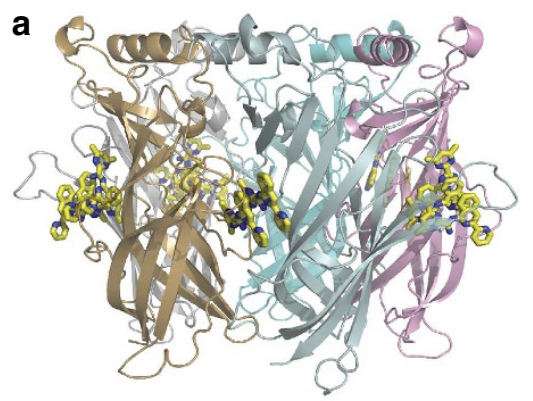

C

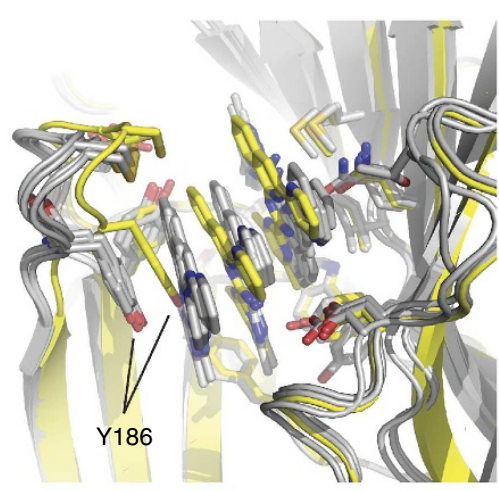

e

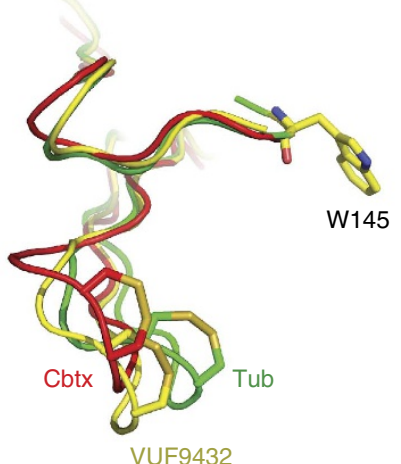

b

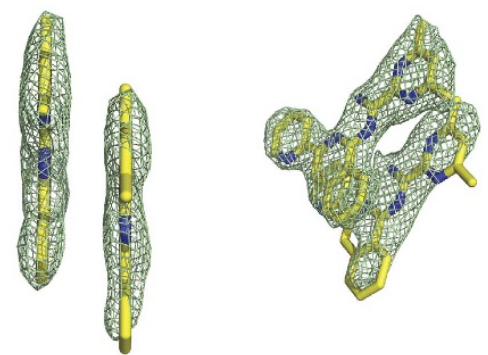

d

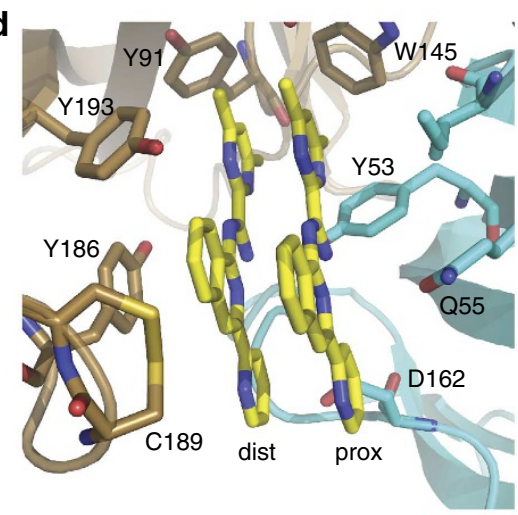

f

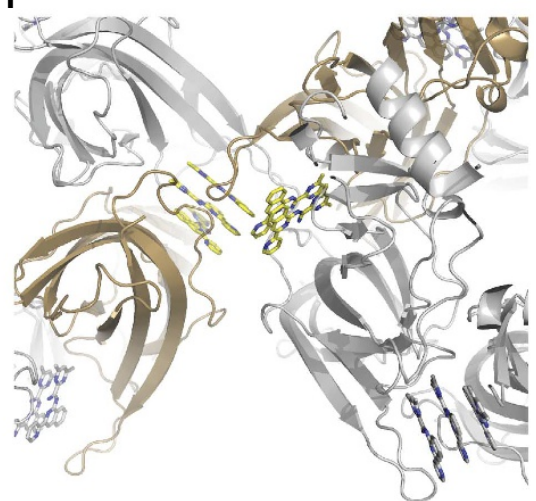

Figure 3 | AChBP can accommodate a double stack of VUF9432. (a) Side view of Ac-AChBP-VUF9432 complex structure, showing two VUF9432 molecules (yellow sticks) in the binding site formed by subunit B (sand) and C (cyan). (b) Electron density map displaying VUF9432 molecules in the ligand-binding site formed by subunit B and $C$ (experimental density contoured at $1 \sigma$ ). (c) Superposition of the four ligand binds site binding triple stacks of VUF9432 (grey cartoon and sticks) and the one binding the double stack of molecule (yellow cartoon and sticks), showing the movement of the Y186 to accommodate the third molecule of the stack. (d) Ball-and-stick representation showing AChBP residues on principal and complementary subunits interacting with the double stack of VUF9432 (colours as in a). (e) Comparison of C-loop opening in AChBP-tubocurarine (Tub, green), AChBP- $\alpha$-cobratoxin (Cbtx, red), AChBP-VUF9432 (the shown C-loop is the one of the ligand-binding site containing two copies of the stack, yellow) complex. Position assumed by the C-loop after VUF9432 binding resembles that in cobratoxin. (f) Interaction of an AChBP-VUF9432 molecule with the symmetry-related molecule that form intermolecular contacts. The open C-loop of subunit B contacts the F-loop of a symmetry-related molecules.

The bound VUF9432 molecules are shown in ball and sticks.

M114 and Q55 at the other end with similar side interactions (Fig. 3d). The major difference for this binding site is caused by the loop C position, which in this case is in an intermediate open state, similar to that found for ligands such as tubocurarine ${ }^{38}$ (PDB:2XYT) and $\alpha$-cobratoxin ${ }^{39}$ (PDB:1YI5) (Fig. 3e).

Interestingly, these contacts resemble those described for the pentameric ring of imidacloprid ${ }^{40,41}$. In that case, the flat aromatic imidazolinic part of the molecule is held in place by face to face stacking with Y186 and upheld by van der Waals contacts with M114. A H-bond connects the Q55 to an oxygen of the nitro group of imidacloprid (Fig. 2d), now held in place by a maximally closed C-loop.
Here, we show that the binding site of AChBP provides space for either one, as in imidacloprid, two or three flat aromatic moieties as seen here for VUF9432, all with similar contacts. This remarkable plasticity is organized primarily by repositioning of the C-loop (Fig. 2d).

Ligand stacking promoted by aromaticity and planarity. To exclude that the unusual configuration of these ligands is a crystallographic artifact, we analysed the binding in several ways. First we confirmed the VUF9432 binding to the ligand-binding site, by mutating interacting amino acids. Mutation of either 
a

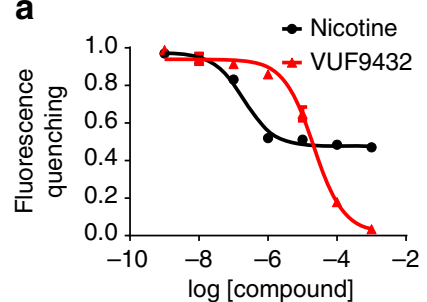

b

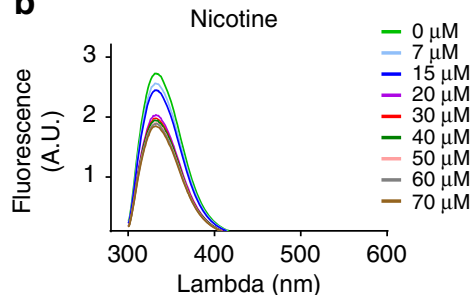

d

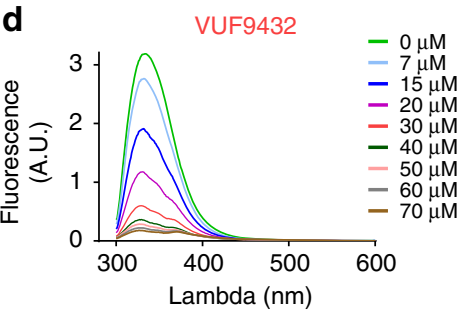

g

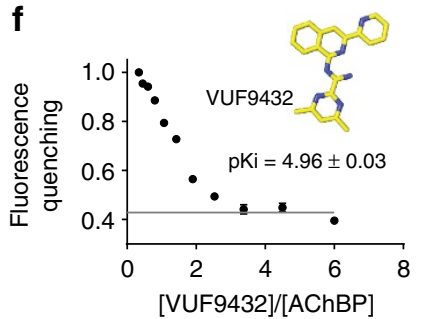

i

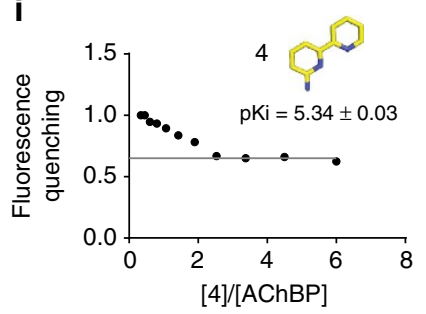

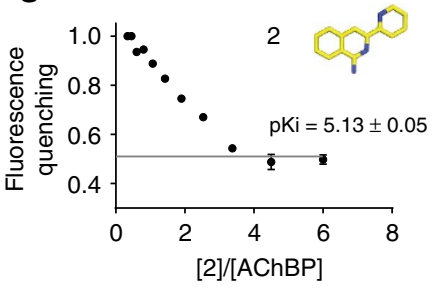

j

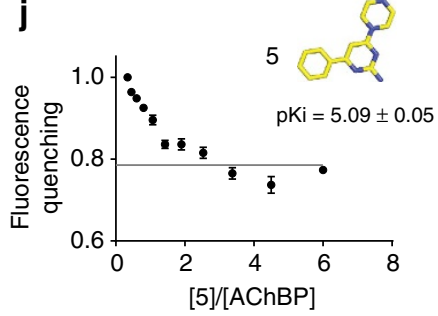

C

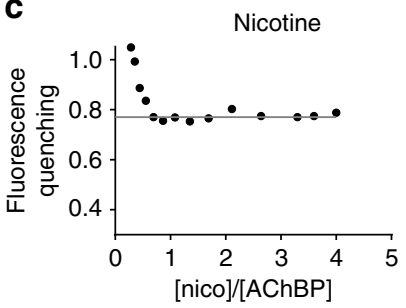

e

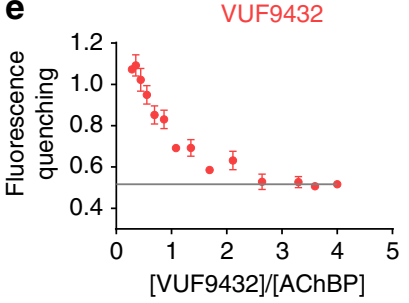

h

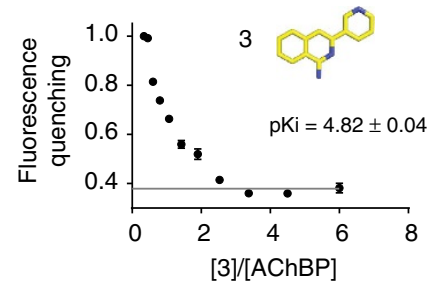

k

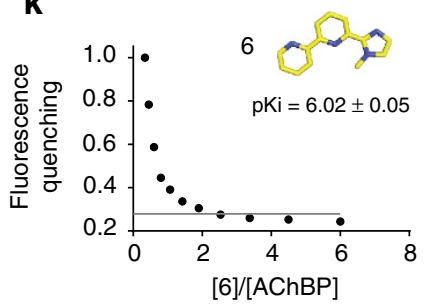

Figure 4 | Aromaticity and planarity determine multiple ligand binding to AChBP. (a) Comparison of affinity of Ac-AChBP for VUF9432 (red curve) and nicotine (black curve) measured with tryptophan fluorescence quenching. Titration of binding sites for nicotine (b,c) and VUF9432 (d,e) on Ac-AChBP by fluorescence quenching. Ac-AChBP at $20 \mu \mathrm{M}$ binding sites was titrated with incremental quantities of ligand. Fluorescence excitation was at $280 \mathrm{~nm}$. Full emission spectra were recorded and maxima were measured over the range $335-345 \mathrm{~nm}(\mathbf{b}, \mathbf{d})$ and plotted versus the nicotine/AChBP (c, black dots) or VUF9432/AChBP (e, red dots) stoichiometric ratio. A straight line was fit through the plateau points (grey line) as described in the methods section. (f-k) Titration of binding sites for the VUF9432 and analogues on Ac-AChBP by fluorescence quenching. Affinity for AChBP (mean \pm s.e.m. measured with radiodisplacement assay) and structure of the ligands (colours as in figure 1) are indicated. AChBP at $20 \mu \mathrm{M}$ was titrated with incremental quantities of ligands as described above (fluorescence maxima plotted as in $\mathbf{c}$ ).

Y186 or Y193 to alanine resulted in the loss of affinity for VUF9432 (Supplementary Fig. S4), confirming, also in solution, the binding to the canonical pocket.

We then tested the stoichiometry of VUF9432 binding to Ac-AChBP in solution, using equilibrium titration. Binding was analysed by the change in the fluorescence emission of W145 of AChBP, which correlates with the binding of agonists or antagonists $^{42}$. Titration of nicotine binding at high protein concentrations showed that the fluorescence quenching reaches a plateau at the stoichiometric ratio ligand/AChBP close to one nicotine molecule bound per protomer (Fig. $4 \mathrm{~b}, \mathrm{c}$ ). In contrast the titration profile of VUF9432 binding does not saturate until a stoichiometry of three ligands per protomer is reached (Fig. 4d,e), confirming the crystallographic data.

We wondered what properties of VUF9432 are necessary for the supramolecular binding. Conformational analysis in the gas phase (Supplementary Fig. S5a) shows that VUF9432 can adopt a series of relatively planar conformations, with minimal rotational freedom of the pyridine ring and of the amidine bond. In the structure a conformation is selected where the pyridine, isoquinoline and pyrimidine rings are coplanar stabilized by a hydrogen bond between the amidine $\mathrm{N} 2$ and the isoquinolinic N4.

We then analysed the original fragment hits for their ligandbinding stoichiometries using fluorescence quenching. Affinity of these fragments for Ac-AChBP was relatively weak (Supplementary Fig. 6, numbers presented as mean pKi in Fig. $4 \mathrm{f}-\mathrm{k}$ ), which meant that we could only use a relative small excess of Ac-AChBP in these experiments. Nevertheless, we could clearly observe that several fragments retain the ability to bind in a three-to-one ratio.

Derivatives,VUF6141 (2) and VUF5954 (3) (Fig. 4g,h), that lack the pyrimidine ring show similar affinity to Ac-AChBP compared with VUF9432, in line with the lack of interaction with Ac-AChBP for this moiety. In computational analysis, both compounds are able to adopt a planar configuration (Supplementary Fig. S5b,c), especially compound 2, where the planar configuration is the most stable, thanks to the $\mathrm{N}$ in position 6 of the pyridine, that prevents clashing between the hydrogen 

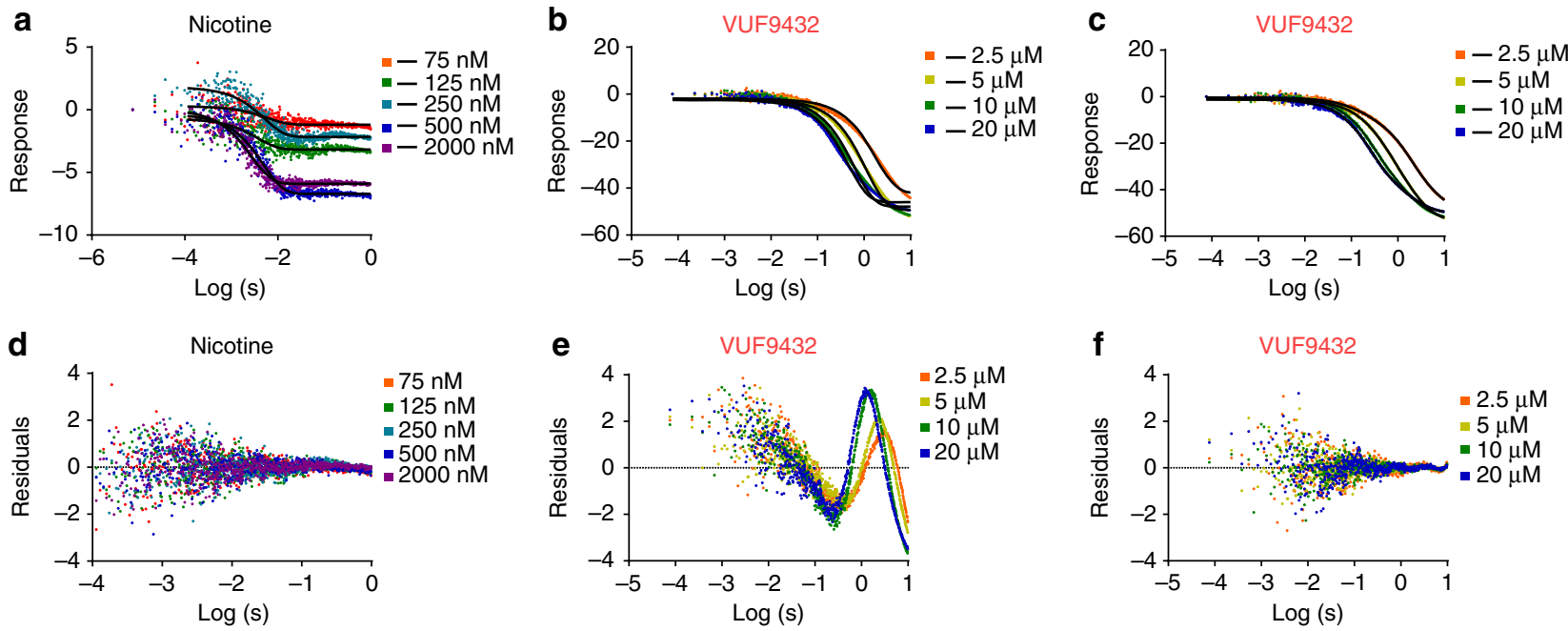

Figure 5 | Binding of VUF9432 is a multistep process. Kinetic studies of ligand association with AChBP. Stopped-flow traces of tryptophan fluorescence quenching on Ac-AChBP binding to nicotine (a) and VUF9432 (b,c). Upper panels show typical traces of observed fluorescence during and after stop page of flow at the indicated ligand concentrations. Panel $\mathbf{d}$ reports the residuals of the fitting for one-step nicotine binding to AChBP. Panels $\mathbf{e}$ and $\mathbf{f}$ report the residuals of the fitting for one (e) or two steps binding (f) of VUF9432 to AChBP, respectively.

atoms of the isoquinolinic and the pyridine rings (Supplementary Fig. S5b,c). Equilibrium fluorescence titration clearly indicates that Ac-AChBP can bind multiple copies of ligand $\mathbf{2}$ and $\mathbf{3}$ per binding site (Fig. 4g,h).

Interestingly, supramolecular binding was also seen for the bi-pyrimidinic compound VUF11370 (4) (Fig. 4i) showing that different aromatic planar molecules can self-assemble in Ac-AChBP. As seen for 2, compound 4 prefers the planar conformation (Supplementary Fig. S5d). In compound VUF10460 (5), a piperazine moiety is present. As seen for nicotine (Fig. 4c) and many other canonical AChBP ligands ${ }^{42}$, this compound induces less fluorescence quenching after binding to $\mathrm{AChBP}$. Equilibrium fluorescence titration indicates the stoichiometry is changed to 1:1, although the affinity for the receptor was maintained (Fig. 4j). This molecule is not fully aromatic resulting in non-planarity of the piperazine moiety (Supplementary Fig. S5e), which most likely prevents the stacking observed in compounds 1-4. Moreover, the compound is slightly basic, which could allow a cation- $\pi$ interaction and an alternative binding mode.

The presence of an imidazolinic moiety like in compound VUF14476 (6) could result in a loss of the binding of multiple copies (Fig. 4k). In compound $\mathbf{6}$, the methyl group attached to the imidazole ring is located at the centre of the molecule (Supplementary Fig. S5f), possibly preventing the formation of stacked molecules. Although there are two methyl groups present on the pyrimidine ring of VUF9432, these are at the edge of the molecule. The slight displacement of the molecules in the stacks may just be enough to ensure the absence of clashes between the hydrogen atoms of the methyl present on the distal and the medial molecules, when located at the edge of the molecule. Even assembling in a similar displaced configuration, the clashes between the hydrogen atoms of the methyl group of $\mathbf{6}$ could probably not be avoided, explaining the one to one stoichiometry to AChBP.

Binding of VUF9432 molecules occurs in steps. An interesting question is whether the three molecules bind as a 'package' or whether they bind sequentially. For this we first analysed the shape of the binding curves of the three molecules in VUF9432 and derivatives 2-4 (Fig. 4a,g-i), some of which may be non-linear.
This non-linearity could reflect differences in affinities for the three VUF9432 sites, resulting in differently shaped binding curves. Alternatively, the effect of binding of the three individual molecules could have differential effects on the tryptophan quenching. One could even imagine that a stack of more than one ligand will receive increased levels of resonance energy transferred from remote aromatic residues, otherwise unaffected by the binding of a single molecule. It will not be trivial to uncouple these possibilities, but it seems likely that the binding event is not a single-step transition of a single package of three ligands into the binding site.

To analyse the binding process in more detail, we used stoppedflow kinetic studies to monitor this assembly process (Fig. 5). Titration of nicotine shows simple association to Ac-AChBP (Fig. $5 \mathrm{a})^{42}$ that can be fitted with a one-step binding model (Fig. 5d). In contrast, the binding profile of VUF9432 cannot be fitted with a simple model (Fig. 5b,c). In practice a two-step fitting of the binding is sufficient to achieve acceptable residuals (Fig. 5e,f), confirming a more complex binding process. In contrast to the proposed binding of bungarotoxin ${ }^{42}$, where the second step is concentration independent and most likely due to a conformational adjustment, for VUF9432 the second step varies with concentration, indicating the stepwise binding of the ligands.

Multiple binding of a fluorescent aromatic molecule to AChBP. We wondered whether different molecules could bind in this manner and searched for planar aromatic molecules. $\mathrm{AO}^{43-45}$ is a fluorescent planar aromatic molecule with increased solubility compared with VUF9432. We found that AO (Fig. 6a) binds the ligand-binding site of AChBP with micromolar affinity ( $\mathrm{pKi}=5.8$, Fig. 6b). An analogue of $\mathrm{AO}, \mathrm{N}$-methylacridinium, was shown to bind in the active site of the enzyme acetylcholinesterase, which also displays affinity for acetylcholine ${ }^{46}$. When AO binding to Ac-AChBP was tested using tryptophan fluorescence quenching at high concentration, we observed that as for VUF9432 the binding has a stoichiometric ratio higher than one (Fig. $6 \mathrm{c}$ ). In agreement with this, titration of Ac-AChBP into an AO solution, Ac-AChBP quenched $\mathrm{AO}$ fluorescence at a stoichiometric ratio of 0.3 (Fig. 6d, black dots). This quenching of $\mathrm{AO}$ by $\mathrm{Ac}-\mathrm{AChBP}$ is inhibited in the presence of an excess of nicotine, confirming that $\mathrm{AO}$ is 
a
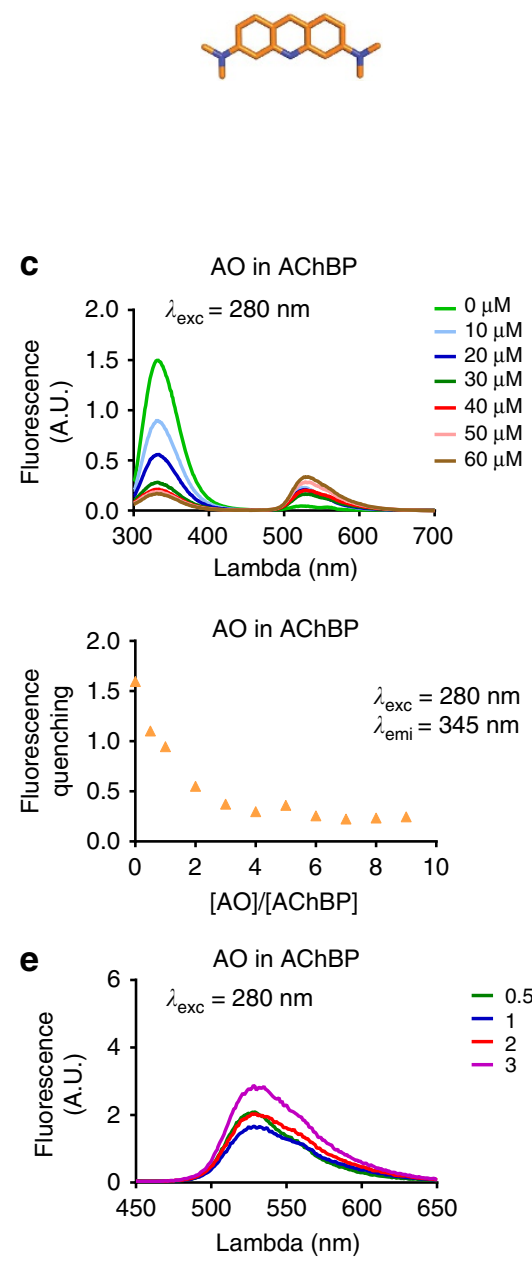

b

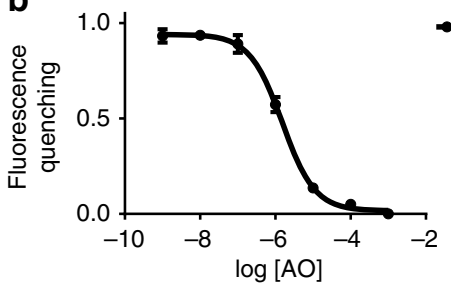

d
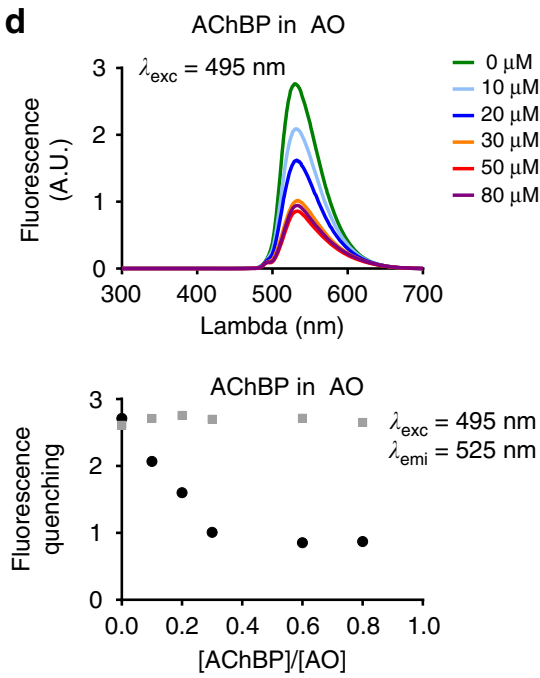

f

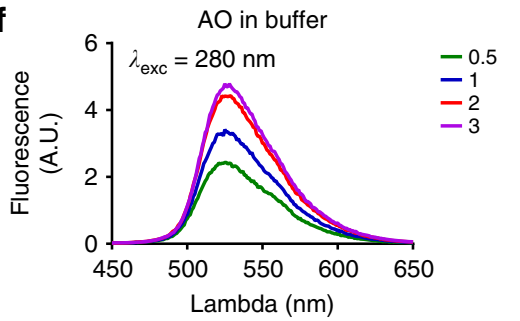

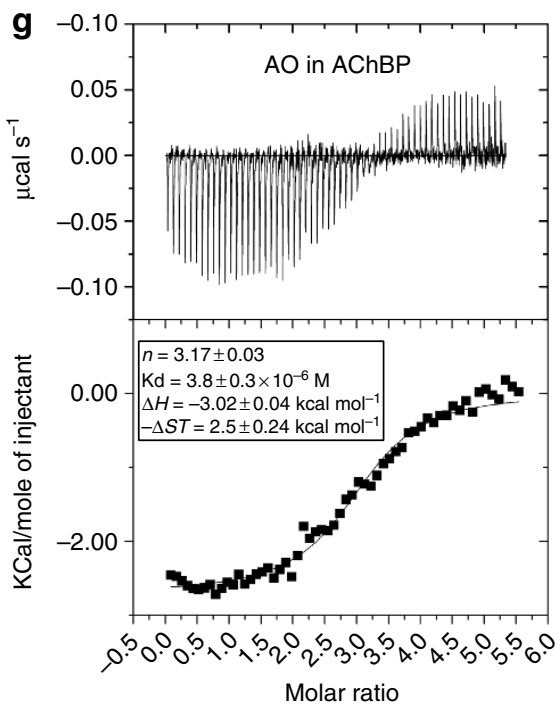

$\mathbf{h}$
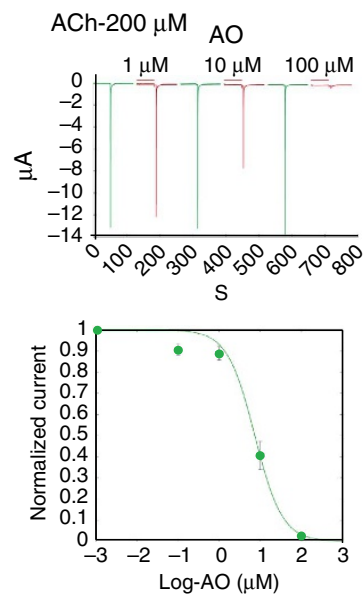

Figure 6 | AO orange mimics the triple stacking ligand. (a) Chemical structure of AO (carbon in orange, nitrogen in blue) (b) Affinity of Ac-AChBP for AO measured with fluorescence quenching (as described in Figure4). (c) Titration of binding sites for AO on Ac-AChBP by tryptophan fluorescence quenching. Ac-AChBP at $20 \mu \mathrm{M}$ was titrated with incremental quantities of AO. Fluorescence excitation was at $280 \mathrm{~nm}$ and full emission spectra were collected between 300 and $700 \mathrm{~nm}$, and emission maxima were measured over the range 335-345 nm and plotted versus the AO/AChBP stoichiometric ratio. (d) Titration of binding sites for AO on Ac-AChBP by AO fluorescence quenching. AO at $100 \mu \mathrm{M}$ was titrated with incremental quantities of $A C h B P$ in presence (grey dots) or in absence of $1 \mathrm{mM}$ nicotine (black dots). Fluorescence excitation was at $495 \mathrm{~nm}$ and full emission spectra were collected between 300 and $700 \mathrm{~nm}$ : emission maxima were measured over the range 525-535 nm and plotted versus the AO-AChBP stoichiometric ratio. Comparison of the fluoresce emission of free $\mathrm{AO}(\mathbf{f})$ or AChBP-AO complex (e) at stoichiometric ratio AO-AChBP of 0.5 (green curve), 1 (blue curve), 2 (red curve) and 3 (purple curve). (g) Calorimetric data of AO binding to AChBP. Top panel shows the raw heat measured over a series of $\mathrm{AO}$ injections $(500 \mu \mathrm{M})$. Each heat signal is integrated and shown as data point in the bottom panel. Data points were fitted to a model describing a single set of binding sites and best-fit parameters for AO binding were calculated using least-squares fitting. (h) Acridine orange inhibits half of ACh-evoked currents at $\alpha 7 \mathrm{nAChR}$ receptors expressed in oocytes at concentrations around $10 \mu \mathrm{M}$ and gives almost full inhibition of the current at $100 \mu \mathrm{M}$.

binding competitively to the binding pocket of Ac-AChBP (Fig. 6d, grey dots).

The formation of the AO stack in Ac-AChBP should determine differences in the emission spectra of the molecules as seen for aromatic $\pi-\pi$ stacking in organic aromatic cages ${ }^{47}$. The fluorescence spectra of $\mathrm{AO}$ in complex with Ac-AChBP and those free in solution were compared. Emission at $525 \mathrm{~nm}$ $\left(\pi-\pi^{*} \text { transition }\right)^{43}$ of the complexed acridine moiety is quenched in the presence of Ac-AChBP due to the interaction of $\mathrm{AO}$ with the aromatic environment. During the titration the intensity at $525 \mathrm{~nm}$ increases without further shifts (Fig. 6f), as expected ${ }^{47}$.

$\mathrm{AO}$ is highly soluble allowing us to use isothermal titration calorimetry (ITC) to follow its binding to AChBP. Our data indicate that $\mathrm{AO}$ binds to $\mathrm{Ac}-\mathrm{AChBP}$ with a stoichiometric ratio of 3-4, with a $\mathrm{Kd}$ of $1.86 \mu \mathrm{M}$ and that the sequential binding is enthalpically favoured (Fig. 6g). Fitting of the experimental data to a model indicates the existence of three events of $\mathrm{AO}$ binding per ligand-binding domain of Ac-AChBP. This confirms that other planar molecules can bind to the Ac-AChBP ligand-binding site in a similar manner to VUF9432. Interestingly, when $\mathrm{AO}$ was tested in electrophysiology experiments on $\alpha 7 \mathrm{nAChR}$-expressing oocytes, it behaved as competitive inhibitor with an IC50 of $7.3 \mu \mathrm{M}$, showing an affinity similar to the one measured for Ac-AChBP (Fig. 6h). Thus, ligands with the potential of supramolecular binding can be relevant for drug design on nAChRs. 


\section{Discussion}

To our knowledge Ac-AChBP is the first example of a target protein binding a triple $\pi-\pi$ stack of ligand molecules. In this paper, we prove that Ac-AChBP binds VUF9432 (Figs 1-5), fragments 2, 3, 4, and $\mathrm{AO}$ with stoichiometry higher than one (Figs 4 and 6). All these compounds address the canonical-binding site of Ac-AChBP and behave as competitive binders (Figs 1, 4 and 6). Aromaticity and planarity of the molecule positively influence the super-stoichiometry (Fig. 4 and Supplementary Fig. S5).

VUF9432 is not very soluble and binds only weakly to $\alpha 7$ nAChR. When tested in electrophysiology experiments, we did not observe any effect of VUF9432 on $\alpha 7$ nAChR expressed in oocytes, probably due to the low solubility (data not shown). However, we identified AO as a molecule that binds AChBP with similar binding properties and stoichiometry. Interestingly, this highly soluble ligand has robust inhibitory properties on nAChR in oocytes, showing the potential for exploitation of these types of ligand binding.

The crystal structure of VUF9432 in Ac-AChBP allows analysis of the supramolecular binding mode. The three molecules of VUF9432 stack in a parallel displaced orientation. The latter was reported to be one of the lowest energetic conformations that such a stack can acquire ${ }^{35}$. In addition several edge-to-face interactions are observed, contributing to the binding properties.

The non-linearity of the binding curves of VUF9432, compound 2-4 and AO, the stopped-flow analysis of VUF9432 binding to Ac-AChBP and the ITC measurement of AO binding to Ac-AChBP suggest that likely the stacking of the molecules is happening in situ and not as preassembled stack (Figs 4-6). However, we cannot exclude other scenarios. Stopped-flow fluorescence measurement of the binding of $\alpha$-bungarotoxin to Ac-AChBP was shown to not be a one-step event ${ }^{43}$. This result was interpreted, suggesting multiple binding modes of the toxin to the binding site of Ac-AChBP. As postulated for the toxin also a stack of VUF9432 could just bind preassembled and have multiple binding modes.

Conformational search performed in gas phase would suggests that this unusual stacking is not present for VUF9432 in solution and that the assembly in situ at the protein-binding site would be energetically more favoured. In particular, the dipole on VUF9432 would disfavour the parallel stacking that is observed for the distal and medial copies of the compound (Figs 1-3). Hence, it is clear that interactions with Ac-AChBP could contribute to stabilize these configurations and that each VUF9432 molecule orients in the ligand-binding site according to the electrostatic environment of the protein rather than their individual local dipole moment.

Entropic factors hamper the intentional creation of parallel $\pi-\pi$ stacks of molecules. 'Tweezer' molecules have been used to stabilize columns of aromatic molecules ${ }^{48}$. Also, reconstitution of a discrete columnar stack of aromatic molecules has been achieved using box-shaped coordination cage ${ }^{47}$. Here, we show that a protein can enable the formation of a $\pi-\pi$ stack of molecules. The stacking is stabilized by the contribution of the aromatic side chains of the ligand-binding site. Although $\pi-\pi$ interactions are thought to be weak ${ }^{35}$, they have been recognized to have an important role in the folding and in the thermal stability of proteins and the binding to ligands.

The binding site in Ac-AChBP is closely related to that of other cys-loop receptors, where specificity for different ligands is provided by amino-acid changes in the loops $\mathrm{A}-\mathrm{C}$ and $\mathrm{D}-\mathrm{F}$. The remarkable plasticity of the ligand-binding site, allowing the binding of differently sized ligands in cys-loop receptors, is tuned by the positioning of the C-loop and has a pivotal role in dictating the dimension of the stack. Recently, Ac-AChBP was found to be capable of accommodating two molecules of acetylcholine it its binding sites, although relative occupancies are very different between the two molecules ${ }^{49}$ (PDB: 2XZ5). Here, we show that the binding site offers a variety of binding possibilities, by simple repositioning of the C-loop (Figs 2 and 3) to accommodate either one, two or three ligand molecules (Fig. 2), with relatively little adjustment in the interactions.

The binding site AChBP was recently shown to accommodate in situ Huisgen cycloaddiction 'click' reactions ${ }^{50}$. Thus, these binding sites can serve as a reaction vessel for chemical reactions. In combination with the ability to promote supramolecular selfassembly, this binding site provides a unique environment for novel chemistry, which can be further tuned by amino-acid variations of loops $\mathrm{A}-\mathrm{F}$, varying the properties of the binding pocket.

Multiple binding in a specific manner within a ligand-binding site provides a novel paradigm for ligand binding. Aggregation of molecules and the presence of multiple independent binding sites have been discussed in the area of drug design previously ${ }^{51}$ However, the interdependent binding required to make a ligand stack is different. It provides specific opportunities as well as novel challenges. Clearly, it will become important to take the stacking possibility into account when analysing ligand binding. Stoichiometry analysis will be important, in particular when considering binding profiles that could potentially provide this type of stacking. Meanwhile, one can see this as a novel opportunity, providing previously unexplored avenues for drug design. Not necessarily trivial, as large flat molecules have obvious disadvantages in design strategies compared with threedimensional fragments ${ }^{51}$, but definitely a new and unexplored opportunity, where a ligand induces different opening of a ligandbinding site while establishing the same interactions with the target protein. In addition, the growing literature on 'tweezer' molecules ${ }^{48}$ and on biocatalytic induction of supramolecular order $^{52}$ may well prove helpful in exploring the possibilities of purposely designing stacks of ligands in proteins.

\section{Methods}

Proteins. Untagged wild-type (wt) and mutants Ac-AChBPs were purified from Sf21 insect cells as previously described ${ }^{19}$. The complementary DNAs for the Ac-AChBP mutants, Ac-AChBP Y186A and Ac-AChBP Y193A, were generated using Quik Change Mutagenesis following the manufacturer's protocols. Mutant proteins were expressed from baculovirus in Sf21 cells. SHS5Y neuroblastoma cell membranes were used as source of $\alpha 7 \mathrm{nAChR}$ in radiodisplacement assays ${ }^{15}$.

Conformational search. A stochastic conformational search was performed using MOE (version 2011.10, Chemical Computing Group Montreal, Canada), using default settings.

Crystallization. The VUF9432-Ac-AChBP complex was formed by mixing the protein at $3.5 \mathrm{mg} \mathrm{ml}^{-1}$ with $1 \mathrm{mM}$ VUF9432 and incubating on ice for $1 \mathrm{~h}$. Cocrystals were grown using the vapour diffusion method in a solution consisting of $0.2 \mathrm{M} \mathrm{Li}_{2} \mathrm{SO}_{4}$ and $0.8 \mathrm{M}$ ammonium sulphate in MMT buffer $(\mathrm{pH} 8.0)$ at $19^{\circ} \mathrm{C}$ Crystals were cryoprotected in mother liquor supplemented with $20 \%$ glycerol and flash-frozen in liquid nitrogen.

Structure solution and refinement. Data were collected on beamline PX1 at the SLS (Switzerland), and processed using iMOSFLM/SCALA software ${ }^{55}$. The VUF9432-Ac-AChBP cocrystal was in space group $\mathrm{P} 2_{1}$ and diffracted to a resolution of $2.4 \AA$. The structure was solved by molecular replacement using PHASER $^{53}$ and the Ac-ACHBP-HEPES structure (2BR7) (ref. 19) as model. Iterative structure refinement was performed using REFMAC ${ }^{54}$ from the CCP4 suite $^{56}$ or BUSTER ${ }^{57}$. Non-crystallographic symmetry restraints (NCSR) were maintained during refinement, using local NCS restraints as implemented in REFMAC or local structure similarity restraints in BUSTER. One TLS group per chain was used in refinement, and both the X-ray weight and B-factor restraint weight were optimized using a local version of the PDB_REDO script ${ }^{58}$. Ligands and water molecules were built in the final stages of refinement. Validation, performed using molprobity ${ }^{59}$ and PDB_REDO, identified $0 \%$ of Ramachandran outliers and $2 \%$ of poor rotamers (overall score: 99 th percentile). Distances, angles and buried 
surface areas were measured in Pymol (DeLano Scientific, LLC). Values and errors in text refer to the average over the four ligand-binding sites with three ligands bound. C-loop opening was calculated measuring the distance between $\mathrm{N} \varepsilon$ of W145 and $\mathrm{S} \gamma$ of Cys 189. Tilting angles of aromatic planes was obtained by calculating the angle between the normal to the planes and averaged over four ligand-binding sites.

Ligand radiodisplacement assays. Competition binding assays were performed with Ac-AChBP (wt or mutants) in buffer (PBS, $20 \mathrm{mM}$ Tris, $\mathrm{pH}$ 7.4/0.05\% Tween) in a final assay volume of $100 \mu \mathrm{l}$ in Optiplates (PerkinElmer Life Science, Inc., USA). Ligands were added at $10^{-3}-10^{-11} \mathrm{M}$. Radioligand, $\left[{ }^{3} \mathrm{H}\right]$ epibatidine (PerkinElmer, specific activity $\sim 56 \mathrm{Ci} \mathrm{mmol}^{-1}$ ), was added at $2.25 \mathrm{nM}$. The amount of protein was chosen such that we obtained a counting window in the displacement curve of $<5 \%$ of the total amount of radioligand, generally $2-20 \mathrm{ng}$. Copper His-Tag PVT SPA beads (PerkinElmer) were added at $2 \mathrm{mg} \mathrm{ml}^{-1}$ final concentration. Plates were incubated at room temperature under continuous shaking, protected from light, for $1.5 \mathrm{~h}$. SPA beads were allowed to settle for $3 \mathrm{~h}$ in the absence of light before counting. The label-bead complex was counted in a Wallac Trilux 1450 Microbeta (PerkinElmer).

Binding assays with $\alpha$-bungarotoxin- ${ }^{125}$ I] (IBTX) were performed as above at $1.8 \mathrm{nM}$ IBTX.

Binding assays with the human $\alpha 7$ receptor were performed as above, but without the SPA beads, as a filtration assay. Human neuroblastoma cells (SHSY5Y) expressing human $\alpha 7 \mathrm{nAChRs}$ (from Christian Fuhrer, Department of Neurochemistry, Brain Research Institute, Zurich) were cultured. The cells were washed three times, and pelleted aliquots were stored frozen at $-80^{\circ} \mathrm{C}$. Before use the cells were resuspended in ice-cold buffer and sonicated. $\left[{ }^{3} \mathrm{H}\right]$ MLA (American Radiolabeled Chemicals, Inc., specific activity $\sim 100 \mathrm{Ci} \mathrm{mmol}^{-1}$ ) was used at a final concentration of $2 \mathrm{nM}$. Bound radioligand was collected on $0.3 \%$ polyethyleneimine-pretreated Unifilter-96 GF/C filters (PerkinElmer). Plates were washed with ice-cold $50 \mathrm{mM}$ Tris buffer at $\mathrm{pH}$ 7.4. After drying the filters, scintillation fluid (MicroScint, PerkinElmer) was added and the radioactivity was counted as above.

All radioligand binding data were evaluated by a non-linear, least-squares curve fitting procedure using GraphPad Prism (version 5, GraphPad Software, Inc., San Diego, CA). All data are represented as mean \pm s.e.m. from at least three independent experiments.

Trp and AO fluorescence quenching. Ac-AChBP, $1 \mu \mathrm{M}$ in binding site, was equilibrated with dilution of the ligands $1 \mathrm{~h}$ before fluorescence measurement. Equilibrium fluorescence was monitored using a PheraStar fluorescence plate reader in the 96-well plates. Ac-AChBP was excited at $280 \mathrm{~nm}$, and emission intensity was monitored at $340 \mathrm{~nm}$ with an emission slit of $8 \mathrm{~nm}$. AO was excited at $495 \mathrm{~nm}$ and emission monitored at $525 \mathrm{~nm}$ with an emission slit of $10 \mathrm{~nm}$. Data were normalized, and $\mathrm{pKi}$ values were calculated by fitting to a sigmoidal dose-response curve with GraphPad.

To determine ligand stoichiometries, Ac-AChBP $(20 \mu \mathrm{M}$ in binding site) was titrated with increasing concentration of ligand until $<8 \%$ quenching was apparent. A straight line was fit through the average of the plateau points. For $\mathrm{AO}$ titration by Ac-AChBP, $\mathrm{AO}(100 \mu \mathrm{M})$ was titrated with increasing concentration of protein. When indicated $1 \mathrm{mM}$ nicotine was added to the assay to compete with AO. Full fluorescence spectra were recorded with a QuantaMaster 3 Fluorometer.

Isothermal calorimetry. ITC experiments were performed on a VP-ITC microcalorimeter (Microcal) at $25^{\circ}$ as described ${ }^{17}$. Ac-AChBP used in these experiments was dialysed in PBS, and the AO was solubilized in the same buffer. For a typical experiment, the ligand at a concentration of $0.5 \mathrm{mM}$ was titrated into $20 \mu \mathrm{M}$ Ac-AChBP. Titration of ligand in buffer alone was performed to determine the change in enthalpy caused by the dilution of the ligand and subtracted as background from the actual ligand-binding experiment. Corrected data were analysed using software supplied by the manufacturer and fitted using a non-linear least-squares method to a model describing one set of binding sites.

Stopped-flow kinetics. Stopped-flow measurement were obtained using a $\mathrm{TgK}$ Scientific stopped-flow system (model SF-61DX2). Ac-AChBP was excited at $280 \mathrm{~nm}$, and emission was recorded with a band filters WG320 and VG11. Changes in fluorescence emission intensity were fit to a first- (nicotine) or a second(VUF9432) order equation.

Electrophysiology. To probe effects of VUF9432 and AO at the $\alpha 7$ nAChR receptors, experiments were conducted at Xenopus oocytes expressing the homomeric human $\alpha 7 \mathrm{nAChRs}$. Expression was obtained by intranuclear injection of $10 \mathrm{nl}$ of a solution containing the cDNA encoding for the human $\alpha 7$ subunit at a concentration of $0.2 \mathrm{\mu g} \mathrm{ml}^{-1}$ using an automated injector (roboinject, Multichannelsystems, Germany). Oocyte preparation and injection was done using the standard procedures as previously described ${ }^{60}$. Three or more day later, electrophysiological properties of the cells were assessed using an automated electrophysiological setup (HiClamp, Multichannelsystems, Germany). Recordings electrodes were filled with $3 \mathrm{M} \mathrm{KCl}$ and oocytes superfused with OR2 medium containing in mM: $\mathrm{NaCl} 82.5, \mathrm{KCl} 2.5$, HEPES $5, \mathrm{CaCl}_{2} \cdot 2 \mathrm{H}_{2} \mathrm{O} 1.8, \mathrm{MgCl}_{2} \cdot 6 \mathrm{H}_{2} \mathrm{O} 1$, $\mathrm{pH}$ 7.4. Cells were held at $-80 \mathrm{mV}$, and $\alpha 7$ expression was tested responses by a brief exposure to $1 \mathrm{mM}$ acetylcholine. Cells displaying robust currents were subsequently tested for their sensitivity to the compound.

\section{References}

1. Murray, C. W. \& Rees, D. C. The rise of fragment-based drug discovery. Nat. Chem. 1, 187-192 (2009).

2. Shokat, K. M. A drug-drug interaction crystallizes a new entry point into the UPR. Mol. Cell 38, 161-163 (2010).

3. Potter, A. J. et al. Structure-guided design of alpha-amino acid-derived Pin1 inhibitors. Bioorg. Med. Chem. Lett. 20, 586-590 (2010).

4. Wiseman, R. L. et al. Flavonol activation defines an unanticipated ligandbinding site in the kinase-RNase domain of IRE1. Mol. Cell. 38, 291-304 (2010).

5. Nissen, M. S. et al. Crystal structures of NADH:FMN oxidoreductase (EmoB) at different stages of catalysis. J. Biol. Chem. 283, 28710-28720 (2008).

6. Sine, S. M. \& Engel, A. G. Recent advances in Cys-loop receptor structure and function. Nature 440, 448-455 (2006).

7. Taly, A., Corringer, P. J., Guedin, D., Lestage, P. \& Changeux, J. P. Nicotinic receptors: allosteric transitions and therapeutic targets in the nervous system. Nat. Rev. Drug Discov. 8, 733-750 (2009).

8. Karlin, A. Emerging structure of the nicotinic acetylcholine receptors. Nat. Rev. Neurosci. 3, 102-114 (2002).

9. Changeux, J. P. \& Taly, A. Nicotinic receptors, allosteric proteins and medicine. Trends Mol. Med. 14, 93-102 (2008).

10. Thompson, A. J., Lester, H. A. \& Lummis, S. C. The structural basis of function in Cys-loop receptors. Q Rev. Biophys. 43, 449-499 (2010).

11. Arneric, S. P., Holladay, M. \& Williams, M. Neuronal nicotinic receptors: a perspective on two decades of drug discovery research. Biochem. Pharmacol. 74, 1092-1101 (2007).

12. Dwoskin, L. P. et al. Nicotinic receptor-based therapeutics and candidates for smoking cessation. Biochem. Pharmacol. 78, 732-743 (2009).

13. Sine, S. M. The nicotinic receptor ligand binding domain. J. Neurobiol. 53, 431-446 (2002).

14. Xiu, X., Puskar, N. L., Shanata, J. A., Lester, H. A. \& Dougherty, D. A. Nicotine binding to brain receptors requires a strong cation-pi interaction. Nature 458, 534-537 (2009).

15. Smit, A. B. et al. A glia-derived acetylcholine-binding protein that modulates synaptic transmission. Nature 411, 261-268 (2001).

16. Brejc, K. et al. Crystal structure of an ACh-binding protein reveals the ligand-binding domain of nicotinic receptors. Nature 411, 269-276 (2001).

17. Celie, P. H. et al. Crystal structure of acetylcholine-binding protein from Bulinus truncatus reveals the conserved structural scaffold and sites of variation in nicotinic acetylcholine receptors. J. Biol. Chem. 280, 26457-26466 (2005).

18. Hansen, S. B. et al. Structural characterization of agonist and antagonist-bound acetylcholine-binding protein from Aplysia californica. J. Mol. Neurosci. 30, 101-102 (2006).

19. Celie, P. H. et al. Crystal structure of nicotinic acetylcholine receptor homolog AChBP in complex with an alpha-conotoxin PnIA variant. Nat. Struct. Mol. Biol. 12, 582-588 (2005).

20. Ulens, C. et al. Structural determinants of selective alpha-conotoxin binding to a nicotinic acetylcholine receptor homolog AChBP. Proc. Natl Acad. Sci. USA 103, 3615-3620 (2006)

21. Rucktooa, P., Smit, A. B. \& Sixma, T. K. Insight in nAChR subtype selectivity from AChBP crystal structures. Biochem. Pharmacol. 78, 777-787 (2009).

22. Celie, P. H. et al. Nicotine and carbamylcholine binding to nicotinic acetylcholine receptors as studied in AChBP crystal structures. Neuron 41, 907-914 (2004).

23. Billen, B. et al. Molecular actions of smoking cessation drugs at alpha4beta2 nicotinic receptors defined in crystal structures of a homologous binding protein. Proc. Natl Acad. Sci. USA 109, 9173-9178 (2012).

24. Unwin, N. Refined structure of the nicotinic acetylcholine receptor at $4 \mathrm{~A}$ resolution. J. Mol. Biol. 346, 967-989 (2005).

25. Dellisanti, C. D., Yao, Y., Stroud, J. C., Wang, Z. Z. \& Chen, L. Crystal structure of the extracellular domain of nAChR alphal bound to alpha-bungarotoxin at 1.94 A resolution. Nat. Neurosci. 10, 953-962 (2007).

26. Hilf, R. J. \& Dutzler, R. X-ray structure of a prokaryotic pentameric ligandgated ion channel. Nature 452, 375-379 (2008).

27. Bocquet, N. et al. X-ray structure of a pentameric ligand-gated ion channel in an apparently open conformation. Nature 457, 111-114 (2009).

28. Li, S. X. et al. Ligand-binding domain of an alpha7-nicotinic receptor chimera and its complex with agonist. Nat. Neurosci. 14, 1253-1259 (2011).

29. Hibbs, R. E. \& Gouaux, E. Principles of activation and permeation in an anionselective Cys-loop receptor. Nature 474, 54-60 (2011).

30. de Kloe, G. E., Bailey, D., Leurs, R. \& de Esch, I. J. Transforming fragments into candidates: small becomes big in medicinal chemistry. Drug Discov. Today 14, 630-646 (2009). 
31. Boyd, S. \& de Kloe, G. E. Fragment library design: efficiently hunting drugs in chemical space. Drug Discov. Today Technol. 7, e173-e180 (2010).

32. Kool, J. et al. High-resolution bioactivity profiling of mixtures toward the acetylcholine binding protein using a nanofractionation spotter technology. J. Biomol. Screen 16, 917-924 (2011).

33. de Kloe, G. E. et al. Online parallel fragment screening and rapid hit exploration for nicotinic acetylcholine receptors. Med. Chem. Commun. 2, 590-595 (2011).

34. van Muijlwijk-Koezen, J. E., Timmerman, H., Link, R., van der Goot, H. \& AP, I. J. A novel class of adenosine A3 receptor ligands. 1.3-(2-Pyridinyl) isoquinoline derivatives. J. Med. Chem. 41, 3987-3993 (1998).

35. Meyer, E. A., Castellano, R. K. \& Diederich, F. Interactions with aromatic rings in chemical and biological recognition. Angew. Chem. Int. Ed. 42, 1210-1250 (2003).

36. Hansen, S. B. et al. Structures of Aplysia AChBP complexes with nicotinic agonists and antagonists reveal distinctive binding interfaces and conformations. EMBO J. 24, 3635-3646 (2005).

37. Edink, E. et al. Fragment growing induces conformational changes in acetylcholine-binding protein: a structural and thermodynamic analysis. J. Am. Chem. Soc. 133, 5363-5371 (2012).

38. Brams, M. et al. A structural and mutagenic blueprint for molecular recognition of strychnine and d-tubocurarine by different cys-loop receptors. PLoS Biol. 9, e1001034 (2011).

39. Bourne, Y., Talley, T. T., Hansen, S. B., Taylor, P. \& Marchot, P. Crystal structure of a Cbtx-AChBP complex reveals essential interactions between snake alpha-neurotoxins and nicotinic receptors. EMBO J. 24, 1512-1522 (2005).

40. Ihara, M. et al. Crystal structures of Lymnaea stagnalis AChBP in complex with neonicotinoid insecticides imidacloprid and clothianidin. Invert. Neurosci. 8, 71-81 (2008).

41. Talley, T. T. et al. Atomic interactions of neonicotinoid agonists with AChBP: molecular recognition of the distinctive electronegative pharmacophore. Proc. Natl Acad. Sci. USA 105, 7606-7611 (2008).

42. Hansen, S. B. et al. Tryptophan fluorescence reveals conformational changes in the acetylcholine binding protein. J. Biol. Chem. 277, 41299-41302 (2002).

43. Feng, X. Z., Lin, Z., Yang, L. J., Wang, C. \& Bai, C. L. Investigation of the interaction between acridine orange and bovine serum albumin. Talanta 47, 1223-1229 (1998).

44. Wang, H., Zhang, W., Dong, X. \& Yang, Y. Thermo-reversibility of the fluorescence enhancement of acridine orange induced by supramolecular self-assembly. Talanta 77, 1864-1868 (2009).

45. Jimenez-Millan, E., Giner-Casares, J. J., Munoz, E., Martin-Romero, M. T. \& Camacho, L. Self-assembly of acridine orange into H-aggregates at the air/water interface: tuning of orientation of headgroup. Langmuir 27, 14888-14899 (2011).

46. Mooser, G. \& Sigmand, D.S. Ligand binding properties of acetylcholinesterase determined with fluorescent probes. Biochemistry 13, 2299-2307 (1974).

47. Yamauchi, Y., Yoshizawa, M., Akita, M. \& Fujita, M. Molecular recognition and self-assembly special feature: discrete stack of an odd number of polarized aromatic compounds revealing the importance of net vs. local dipoles. Proc. Natl Acad. Sci. USA 106, 10435-10437 (2009).

48. Leblond, J. \& Petitjean, A. Molecular tweezers: concepts and applications. Chemphyschem 12, 1043-1051 (2011).

49. Brams, M. et al. Crystal structures of a cysteine-modified mutant in loop D of acetylcholine-binding protein. J. Biol. Chem. 286, 4420-4428 (2011).

50. Grimster, N. P. et al. Generation of candidate ligands for nicotinic acetylcholine receptors via in situ click chemistry with a soluble acetylcholine binding protein template. J. Am. Chem. Soc. 134, 6732-6740 (2012).

51. Hung, A. W. et al. Route to three-dimensional fragments using diversityoriented synthesis. Proc. Natl. Acad. Sci. USA 108, 6799-6804 (2011).

52. Hirst, A. R. et al. Biocatalytic induction of supramolecular order. Nat. Chem. 2, 1089-1094 (2010)
53. McCoy, A. J. et al. Phaser crystallographic software. J. Appl. Crystallogr. 40, 658-674 (2007).

54. Murshudov, G. N., Vagin, A. A. \& Dodson, E. J. Refinement of macromolecular structures by the maximum-likelihood method. Acta Crystallogr. D Biol. Crystallogr. 53, 240-255 (1997).

55. Evans, P. Scaling and assessment of data quality. Acta crystallographica. Sec. D Biol. Crystallogr. 62, 72-82 (2006).

56. Winn, M. D. et al. Overview of the CCP4 suite and current developments. Acta crystallographica. Sec. D Biol. Crystallogr. 67, 235-242 (2011).

57. Global Phasing Ltd.. BUSTER v. 2.8.0. (Global Phasing Ltd., 2011).

58. Joosten, R. P., Joosten, K., Cohen, S. X., Vriend, G. \& Perrakis, A. Automatic rebuilding and optimization of crystallographic structures in the Protein Data Bank. Bioinformatics 27, 3392-3398 (2011).

59. Chen, V. B. et al. MolProbity: all-atom structure validation for macromolecular crystallography. Acta Crystallogr. Sec. D Biol. Crystallogr. 66, 12-21 (2010)

60. Hogg, R. C., Bandelier, F., Benoit, A., Dosch, R. \& Bertrand, D. An automated system for intracellular and intranuclear injection. J. Neurosci. Methods 169, 65-75 (2008)

\section{Acknowledgements}

We thank J. W. Borst and T. Visser for critically reading the manuscript. We thank the staff from SLS PXI and ESRF for assistance during data collection and R. Joosten and A. Perrakis for useful discussions during the refinement and data validation steps. We thank T. Schaer and E. Neveu for their help in the electrophysiological experiments.We thank A. Perrakis, P. Taylor, P. Celie, A. Amore., H. Ovaa, J. Hausmann, J. E. van Muijlwijk-Koeze and C. Verlinde for fruitful discussions. This work was supported by the European Union Seventh Framework Programme under Grant agreement HEALTH-F2 2007-202088 (Neurocypres project) (to T.K.S., A.B.S. and I.J.P.E.) and by TIPharma Grant D2-103 (to T.K.S., A.B.S. and I.J.P.E.).

\section{Author contributions}

The experimental work was performed by M.S., with contribution from P.R. for the structure refinement and from A.F. for the biophysical experiments. G.E.K. and E.S.E. performed the chemical analysis of the compounds by NMR and high-resolution mass spectral analysis, and they also performed their conformational analysis. R.v.E. performed the affinity measurement of the ligands for AChBP and the nAChR receptors. D.B. performed the electrophysiology measurement. M.S., T.K.S. and P.R. planned the work and analysed the results. The paper was written by T.K.S and M.S. with assistance from the other authors.

\section{Additional information}

Accession codes: Atomic coordinates and structure factors for the reported crystal structure have been deposited in the Protein Data Bank with accession code $4 \mathrm{bfq}$.

Supplementary Information accompanies this paper at http://www.nature.com/ naturecommunications

Competing financial interests: The authors declare no competing financial interests.

Reprints and permission information is available online at http://npg.nature.com/ reprintsandpermissions/

How to cite this article: Stornaiuolo, M. et al. Assembly of a $\pi-\pi$ stack of ligands in the binding site of an acetylcholine-binding protein. Nat. Commun. 4:1875 doi: 10.1038/ ncomms2900 (2013).

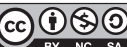

This work is licensed under a Creative Commons AttributionNonCommercial-ShareAlike 3.0 Unported License. To view a copy of this license, visit http://creativecommons.org/licenses/by-nc-sa/3.0/ 